\title{
Expression of Genes Involved in Proanthocyanidin Biosynthesis during Fruit Development in a Chinese Pollination-constant, Nonastringent (PCNA) Persimmon, 'Luo Tian Tian Shi'
}

\author{
Ayako Ikegami and Keizo Yonemori ${ }^{1}$ \\ Laboratory of Pomology, Graduate School of Agriculture, Kyoto University, Sakyo-ku, Kyoto 606-8502, \\ Japan
}

\author{
Akira Kitajima \\ Experimental Farm, Graduate School of Agriculture, Kyoto University, Takatsuki City, Osaka 569- \\ 0096, Japan
}

\author{
Akihiko Sato and Masahiko Yamada \\ Department of Grape and Persimmon Research, National Institute of Fruit Tree Science, Akitsu, \\ Hiroshima 729-2492, Japan
}

AdDitional InDEX wORDS. Diospyros kaki, condensed tannin, astringency, proanthocyanidin biosynthesis, gene expression

\begin{abstract}
Expression patterns of the genes involved in condensed tannin (CT) biosynthesis during fruit development was investigated in a Chinese pollination-constant, nonastringent (PCNA) persimmon (Diospyros kaki Thunb.) 'Luo Tian Tian Shi'. The transcript levels of phenylalanine ammonia-lyase $(P A L)$ and dihydroflavonol reductase $(D F R)$ in 'Luo Tian Tian Shi' were detected at high levels throughout the fruit growth. Chalcone synthase $(C H S)$ and flavonol 3-hydroxylase $(\mathrm{F3H})$ also continued to be transcribed during fruit growth, although their levels decreased earlier than $P A L$ and $D F R$. In contrast, expression levels of these genes declined into undetectable levels at an early stage of fruit development in Japanese PCNA persimmon. In addition, anthocyanidin reductase $(A N R)$, which encodes a key enzyme of the proanthocyanidin biosynthesis, was transcribed at high levels in 'Luo Tian Tian Shi' during fruit growth, but not in Japanese PCNA persimmon. By contrast, the expression of D. kaki serine carboxypeptidase-like protein 1 (DkSCPL1) that was obtained from suppression subtractive hybridization (SSH) analysis between artificially astringency-removed fruit and astringent fruit in a different experiment, declined earlier than the other flavonoid biosynthesis genes in 'Luo Tian Tian Shi', coincident with the termination of the tannin cell development. In the $F_{1}$ progeny of the cross between 'Luo Tian Tian Shi' and Japanese PCNA 'Taishu', similar expression patterns were obtained among segregated PCNA and astringent offspring. These results indicate that Chinese PCNA is different from Japanese PCNA in expression of the genes involved in CT biosynthesis. In conclusion, we clarified that expression of the genes (PAL to $A N R$, but not $S C P L)$ involved in flavonoid biosynthesis was continuous in the Chinese PCNA cultivar, despite the termination of tannin cell development.
\end{abstract}

Japanese persimmon cultivars are classified into four groups depending on the effect of pollination on flesh color and whether the fruit lose astringency at harvest time on the tree: i.e., pollination-constant nonastringent (PCNA); pollination-constant astringent (PCA); pollination-variant nonastringent (PVNA); and pollination-variant astringent (PVA) (Yonemori et al., 2000). Among these cultivars, only PCNA-type fruit terminate tannin accumulation at an early stage of fruit development and the fruit lose astringency naturally on the tree with fruit growth regardless of seedless or seeded (Yonemori and Matushima, 1985, 1987). This trait of astringency-loss in PCNA persimmon is qualitatively inherited and recessive to non-PCNA (PCA, PVNA, and PVA) type. In fact, crosses among PCNA-type cultivars or selections yield only PCNA offspring in $\mathrm{F}_{1}$ generation, however, a cross between PCNA and non-PCNA-type yields only non-PCNA-type offspring (Ikeda et al., 1985; Yamada and Sato, 2002). On the

Received for publication 10 Feb. 2005. Accepted for publication 4 Apr. 2005. We thank Dr. Frederick G. Gmitter for discussion and advice, and critical reading of the manuscript.

'Corresponding author: fax +8175753 6497; e-mail: keizo@kais.kyoto-u.ac. jp. contrary, in non-PCNA-type fruit, tannin accumulation continues during fruit development and the loss of astringency is dependant on the amounts of ethanol and acetaldehyde generated by the seeds (Sugiura and Tomana, 1983; Yonemori and Matsushima, 1985). The ability of the seeds to generate these volatile compounds is high in the PVNA-type, little in the PCA-type, and moderate in the PVA-type fruit. Therefore, PVNA-type fruit with seeds lose astringency completely by coagulation of tannins with acetaldehyde on the tree. The coagulation of tannins is restricted around the seeds in PVA-type cultivars due to limited amounts of volatile compounds, and PCA-type fruit do not lose astringency naturally on the tree as almost no ethanol and acetaldehyde production during their development. The crosses among non-PCNA-type cultivars yield PVNA, PVA, or PCA-type, as the ability of seeds to generate these volatile compounds is quantitatively inherited (Ikeda et al., 1985).

Incorporating the trait of PCNA into new cultivars is one of the most important objectives in breeding projects (Yamada, 1993; Yamada et al., 1994). PCNA-type cultivars are thought to have originated uniquely in Japan, but only six cultivars among more than 1000 persimmon cultivars collected from all regions of Japan were found to be PCNA-type by a nationwide survey 
(Agricultural Research Station, 1912). The genetic diversity of PCNAcultivars was reported to be very narrow due to their young origin (Yamada and Sato, 2002). Recently, however, a PCNA-type, 'Luo Tian Tian Shi', was found in the Luo Tian county of China (Wang, 1982; Wang et al., 1997). This cultivar was demonstrated to have a unique inheritance of the trait of natural astringencyloss; the cross between PCNA 'Luo Tian Tian Shi' and Japanese PCNA 'Taishu' yielded non-PCNA and PCNA-type individuals in $\mathrm{F}_{1}$ generation at the ratio of 22:11 (Ikegami et al., 2004). This result suggests the locus controlling natural astringency-loss in 'Luo Tian Tian Shi' fruit seems to be independent of that in Japanese PCNA persimmons.

Most persimmon fruit accumulate a large quantity of highmolecular-weight tannins consisting of proanthocyanidins into "tannin cells" that are specialized for tannin accumulation. These high-molecular weight tannins are the cause of astringency in persimmon fruit (Porter and Woodruffe, 1984). Proanthocyanidins (PAs), also called condensed tannins (CTs), are synthesized via the flavonoid biosynthetic pathway, and these compounds also include subgroups that are found in most higher plants: the chalcones, flavones, flavonols, and anthocyanins (Marles et al., 2003; Winkel-Shirley, 2001, 2002). The biosynthetic pathway consists of structural genes encoding the enzymes that directly participate in the formation of flavonoids (Fig. 1), as well as regulatory genes that control the transcription of these structural genes. A gene encoding leucoanthocyanidin reductase (LAR), which catalyzes the formation of catechins has been obtained from the tropical forage Desmodium uncinatum (Jacq.) DC. (Tanner et al., 2003). The BANYULS gene in Arabidopsis thaliana (L.) Heynh. has been shown to encode anthocyanidin reductase (ANR), which in turn converts anthocyanidins into epicatechins (Xie et al., 2003). Six different types of regulatory elements [e.g., TFIIIA-like, WD-40-like, WRKY-like, MADS-box-like, myb-like, and bHLH (myc-like)], have been cloned and identified using mutants from A. thaliana (tt1, ttg 1, ttg 2, tt2, tt16,tt2,tt8) and two other species [Hordeum vulgare L. (ant13) and Lotus spp. L. (tan1)] (Marles et al., 2003). However, the biosynthetic pathway involved in the production of CTs is not well understood, although it is known to include the enzyme gallic acid transferase, vacuolar membrane transporters for leucoanthocyanidins and proanthocyanidins, and condensing enzyme (Park et al., 2004).

In a previous study, we observed the termination of expression of all structural genes involved in flavonoid biosynthesis at an early stage of fruit development in Japanese PCNA cultivars (Ikegami et al., 2005). We also isolated ANR (a key enzyme of PA biosynthesis) and DkSCPL1 (a candidate enzyme involved in CT biosynthesis) (Ikegami et al., unpublished) using suppression subtractive hybridization (SSH) (Diatchenko et al., 1996) from artificially astringency-removed fruit on the tree by enclosing them with polyethylene bags containing $10 \mathrm{~mL}$ of $5 \%$ aqueous ethanol solution (Sugiura et al., 1975). In this study investigating expression of these genes involved in CT biosynthesis, we characterized genetic control of tannin accumulation in Chinese PCNA 'Luo Tian Tian Shi', in order to understand basic information of its trait of natural astringency-loss, which will become a promising breeding resource in the near future.

\section{Materials and Methods}

Plant Material. The fruit of Chinese PCNA 'Luo Tian Tian Shi', Japanese PCNA 'Suruga' and PCA 'Kuramitsu', growing in the experimental orchard of Kyoto Univ. in Kyoto, Japan,

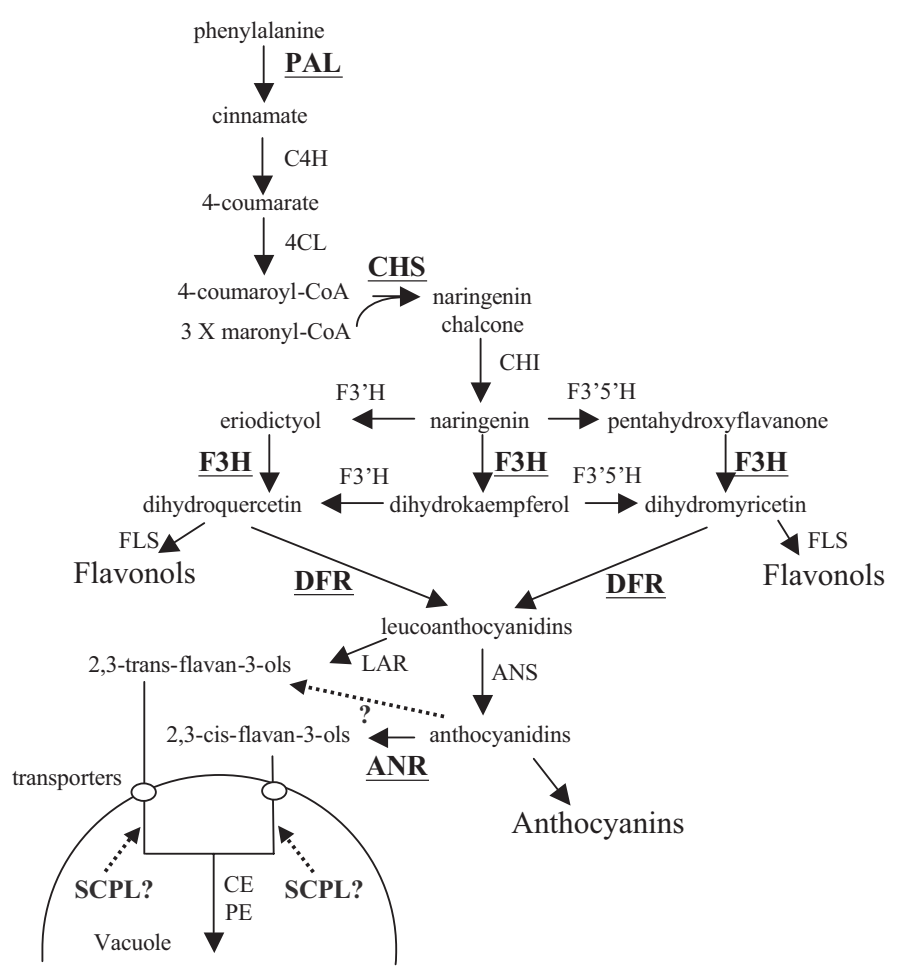

PROANTHOCYANIDINS

Fig. 1. Scheme of the flavonoid biosynthetic pathway. Enzymes in boldface letters are those for which gene expression was analyzed in this study (ANS $=$ anthocyanidin synthase, $\mathrm{ANR}=$ anthocyanidin reductase, $\mathrm{CE}=$ condensing enzyme, $\mathrm{C} 4 \mathrm{H}=$ cinnamate-4-hydroxylase, $\mathrm{CHI}=$ chalcone isomerase, $\mathrm{CHS}=$ chalcone synthase, $4 \mathrm{CL}=4$-coumarate: coenzyme $\mathrm{A}$ ligase, $\mathrm{DFR}=$ dihydroflavonol reductase, $\mathrm{F} 3 \mathrm{H}=$ flavonol 3-hydroxylase, $\mathrm{F}^{\prime}$ ' $\mathrm{H}=$ flavonoid 3' hydroxylase, F3'5 $^{\prime} \mathrm{H}=$ flavonoid 3'5' hydroxylase, FLS = flavonol synthase, $\mathrm{LAR}=$ leucoanthocyanidin reductase, $\mathrm{PAL}=$ phenylalanine ammonia-lyase, $\mathrm{PE}=$ polymerizing enzyme, SCPL = serine carboxypeptidase-like protein). Adapted from Nesi et al. (2001), Bartel and Matsuda (2003), Xie et al. (2003), and Debeaujon et al. (2003). Note that the function of SCPL is putative.

were sampled every 2 weeks from June to Nov. 2003. Fruit of six $F_{1}$ individuals in 310-line derived from the cross between 'Luo Tian Tian Shi' and Japanese PCNA 'Taishu', growing in the Dept. of Grape and Persimmon Research, National Institute of Fruit Tree Science, Hiroshima, Japan, also were sampled on 12 June, 12 July, 3 Aug., and 3 Sept. 2001. We selected three each representatives for PCNA or non-PCNA individuals from 22 PCNA and 11 non-PCNA-type offspring considering average size of the fruit. At least three fruit of each cultivar or selection were weighed and diced, and fixed for tannin cell size measurement. A second set of three fruit were sampled, peeled, and flesh diced, then frozen with liquid nitrogen and stored at $-80{ }^{\circ} \mathrm{C}$ for the RNA isolation.

TANNIN CELL SIZE DETERMination. Small blocks of tissue from the equatorial region of the mesocarp were fixed with $2.5 \%$ glutaraldehyde, containing $0.2 \%$ tannic acid. After the blocks were washed with water, they were macerated in a $0.05 \mathrm{M}$ EDTA solution adjusted to $\mathrm{pH} 10.0$ at $45^{\circ} \mathrm{C}$ for $5 \mathrm{~h}$ by oscillating at $90 \mathrm{rpm}$ according to Letham (1960). A droplet of tannin cells, separated from the parenchyma cells by decanting several times, was placed on a glass slide, and images of the tannin cells taken with a digital camera attached to a light microscope (DP-50; Olympus Corp., Tokyo) were recorded on a computer. The area of 100 tannin cells from each sample was measured with Scion Image, public domain software (Scion Corp., Frederick, Md.). 
ISOLATION OF TOTAL RNA AND GEL BLOT ANALYSIS. Total RNA was isolated from persimmon fruit by the hot borate method (Wan and Wilkins, 1994). The quality of the isolated total RNA was verified on a denatured $1 \%$ (w/v) ethidium bromidestained agarose gel. RNA concentrations were determined spectrophotometrically. Total RNA $(15 \mu \mathrm{g})$ was applied to a denatured agarose gel, and was blotted onto Hybond-N membrane (Amersham Biosciences, Piscataway, N.J.). The blots were hybridized with digoxigenin (DIG)-labeled PCR fragments amplified with gene specific primers for persimmon sequence involved in proanthocyanidin biosynthetic pathway (Table 1). PCR probes were labeled with a PCR-DIG probe synthesis kit (Roche Diagnostics, Basel, Switzerland). After hybridization for $16 \mathrm{~h}$ at $42^{\circ} \mathrm{C}$ with "high SDS" buffer [50 mm phosphate buffer (pH 7.0), $7 \%$ SDS, $50 \%$ formamide, $2 \%$ blocking reagent, $5 \times$ SSC, and $0.1 \% \mathrm{~N}$-lauroylsarcisine], membranes were washed twice in $2 \times \mathrm{SSC}$ containing $0.1 \% \mathrm{SDS}$ at $42{ }^{\circ} \mathrm{C}$ for $5 \mathrm{~min}$ each, followed by washing twice in $0.1 \times \mathrm{SSC}$ containing $0.1 \%$ SDS at $56{ }^{\circ} \mathrm{C}$ for $15 \mathrm{~min}$. Target RNA was detected according to the manufacturer's manual (Roche Diagnostics).

\section{Results}

The development of tannin cells was stopped at the early growth stage of the fruit in July with 'Suruga' and 'Luo Tian Tian Shi', while it continued until the late stage in PCA 'Kuramitsu' (Fig. 2). This means that in both PCNA cultivars tannin synthesis terminated in the early stage, whereas the PCA cultivar continued to synthesize tannins to the late stages.

Seasonal changes in gene expression involved in flavonoid biosynthesis ( $P A L, C H S, F 3 H$, and $D F R$ ) were compared among the three cultivars. Gene transcripts were detected at high levels through the fruit growth stages in the PCA cultivar (Fig. 3). By contrast, very low transcription levels were found in Japanese PCNA fruit from middle to late growth stages. In Chinese PCNA 'Luo Tian Tian Shi', transcription of $C H S$ and $F 3 H$ reduced earlier than in PCA cultivar, but those of $P A L$ and $D F R$ continued relatively high levels throughout the fruit growth. The transcripts of these genes involved in flavonoid biosynthesis in 'Luo Tian Tian Shi' were detected throughout the fruit growth, despite the termination of the tannin cell enlargement (Fig. 2). The expression patterns of these genes in the Japanese PCNA cultivar were different from both the PCA cultivar, and Chinese PCNA 'Luo Tian Tian Shi’.

In addition, previously, we performed SSH analysis to isolate genes involved in CT biosynthesis from fruit that had an artificial astringency-removal treatment on a tree (Ikegami et al., unpublished). Astringency-removal was performed using young fruit of PVA 'Hiratanenashi' by enclosing with polyethylene bags containing $10 \mathrm{~mL}$ of $5 \%$ aqueous ethanol solution as previously reported (Sugiura et al., 1975). This treatment caused tannin coagulation in the fruit and terminated expression of the genes involved in flavonoid biosynthetic pathway such as $P A L, C H S$, and DFR. By $\mathrm{SSH}$ analysis between artificially astringency-removed fruit and astringent fruit, a clone having anthocyanidin reductase (ANR)like sequence, which is known to be one of the key enzymes for PA biosynthesis, was identified. ANR expression in the fruit of 'Luo Tian Tian Shi' was relatively high, despite the termination of the tannin cell enlargement (Fig. 4). Expression levels of another SSH fragment having the sequence of serine carboxypeptidaselike protein (DkSCPL1) that may have the function related to CT accumulation, were also evaluated (Fig. 4). Accumulation of the
Table 1. Sequences of the primers used for digoxigenin (DIG) probe synthesis. The primer pairs were prepared for phenylalamine ammonia-lyase (PAL), chalcone synthase (CHS), flavonol 3-hydroxylase $(\mathrm{F} 3 \mathrm{H})$, and dihydroflavonol reductase (DFR) as described previously (Ikegami et al., 2005). The primers for anthocyanidin reductase (ANR) and Diospyros kaki serine carboxypeptidase-like protein (DkSCPL1) were designed according to the sequence of persimmon (Ikegami et al., unpublished).

\begin{tabular}{ll} 
Gene & primers \\
\hline \hline PAL & F5'-TGGATAATACCCGACTAGCAATC-3' \\
& R5'-CCTTGAAGCCATAGTCCAAGCT-3' \\
& \\
CHS & F5'-CGCTGCCGCTATAATTGTTG-3' \\
& R5'-TGGAATGTAAGGCCCACTTCA-3' \\
& F5'-GATAAGTTGATGGGCCTGGCCTGCAAAT-3' \\
& R5'-AGGTCTGGTTGCGGGCATTTCGGGTAGA-3' \\
& F5'-CAATGGAGTTCTGGGCATCA-3' \\
DFR & R5'-TCCGGTCATTCTTGGAGTAGA-3' \\
& \\
ANR & F5'-GCTGAGTCAAGCAGCTTGGA-3' \\
& R5'-ATGGCAAGACCCATACTGCT-3' \\
& \\
DkSCPL1 & F5'-GCGTGGCAGTGCTTCAGGGA-3' \\
& R5'-GGAGTCCGTGAACTCTGCTT-3' \\
\hline \hline
\end{tabular}

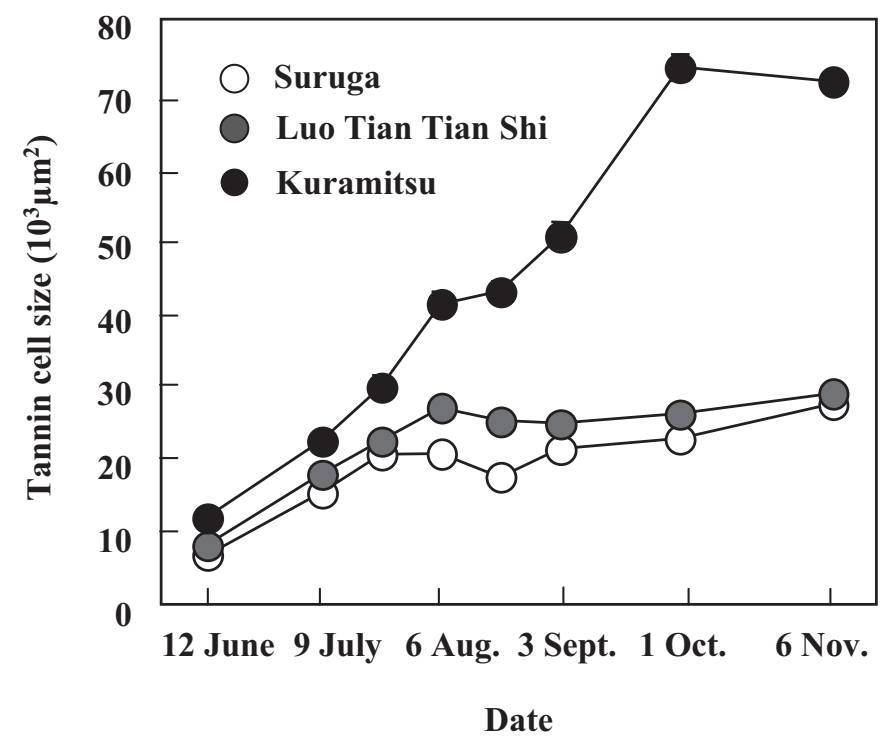

Fig. 2. Changes in tannin cell size during fruit development of Chinese pollinationconstant, nonastringent (PCNA) 'Luo Tian Tian Shi'; Japanese PCNA 'Suruga'; and pollination-constant, astringent (PCA) 'Kuramitsu'.

transcripts of DkSCPL1 in 'Luo Tian Tian Shi' fruit decreased coincidentally with the termination of tannin cell enlargement, unlike $A N R$ and other flavonoid pathway genes.

In the $\mathrm{F}_{1}$ offspring derived from the cross between 'Luo Tian Tian Shi' and Japanese PCNA 'Taishu', PCNA-type and non-PCNA-type offspring segregated as previously reported (Ikegami et al., 2004). In the present study, we selected three individuals from each type of offspring. Segregated PCNA-type (310-17, 36 and 41) showed PCNA trait of early termination of 

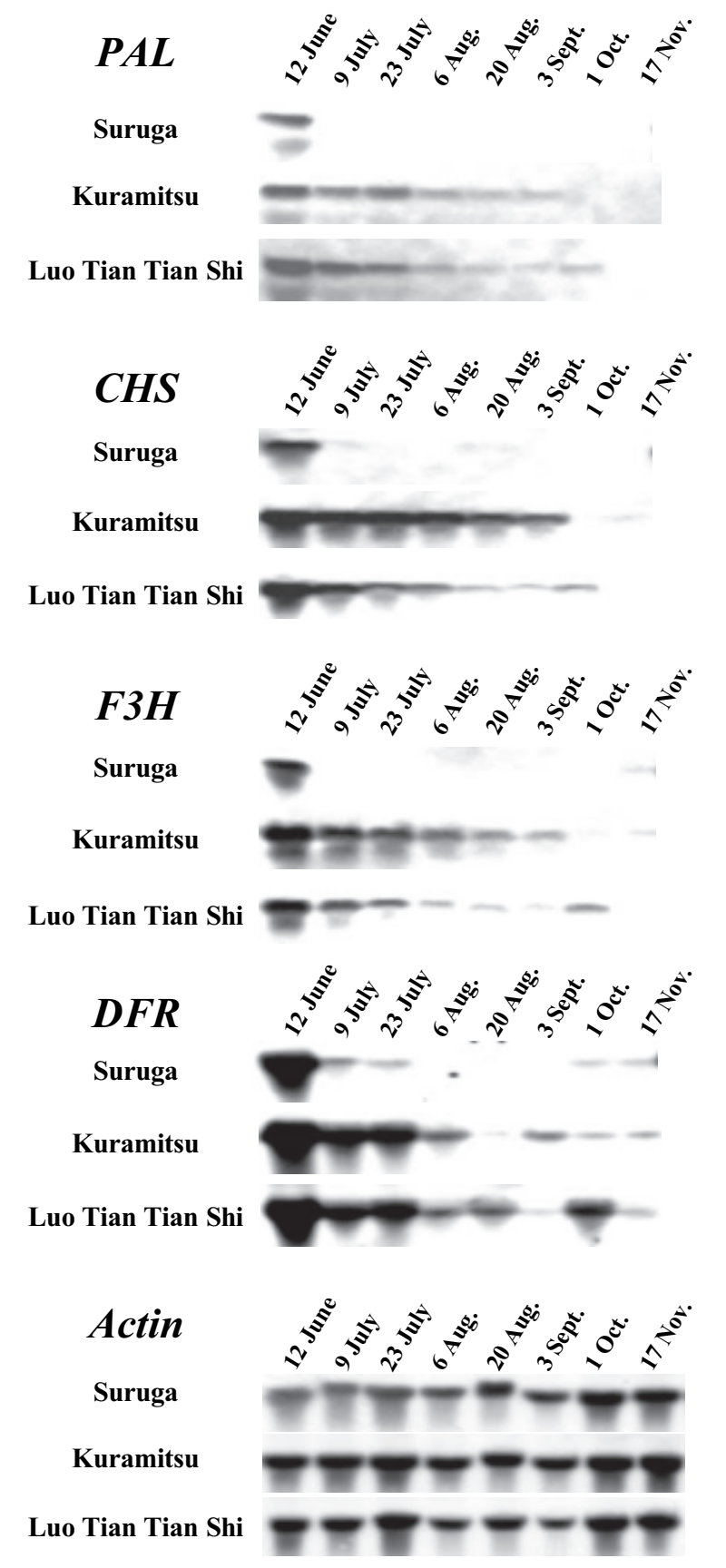

Fig. 3. Seasonal expression of the genes involved in the flavovnoid biosynthetic pathway $(P A L=$ phenylalanine ammonia-lyase, $C H S=$ chalcone synthase, $F 3 H=$ flavonol 3-hydroxylase, $D F R=$ dihydroflavonol reductase) during fruit development of Chinese pollination-constant, nonastringent (PCNA) 'Luo Tian Tian Shi'; Japanese PCNA 'Suruga'; and pollination-constant, astringent (PCA) 'Kuramitsu'. RNA gel blots are of total RNA (15 $\mu \mathrm{g})$ from persimmon fruit samples taken from June to November during fruit development. Japanese pear (Pyrus pyrifolia Nakai) 'Housui' cDNA clone for actin was the probe used for a control.

tannin cell development, while non-PCNA type (310-43, 108, and 119) continued tannin cell enlargement to the late growth stage (Fig. 5). In these progenies, gene expression (PAL, CHS, $F 3 H$, and $D F R$ ) involved in flavonoid biosynthetic pathway and ANR showed similar patterns (Fig. 6) as cultivars described above. On the contrary, transcript levels of DkSCPL1 in PCNA offspring were much less than those of non-PCNA ones on 12 July and 3 Aug.
ANR

122792362031711626617 June July Aug. Sept. $\overline{\text { Oct. }} \overline{\text { Nov. }}$

Suruga

Kuramitsu

Luo Tian Tian Shi

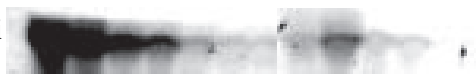

DkSCPL1

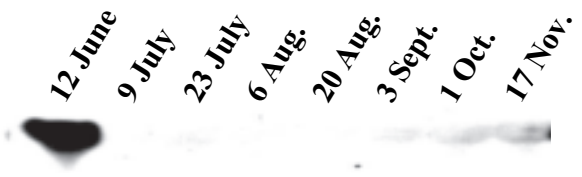

Kuramitsu

\section{Luo Tian Tian Shi}

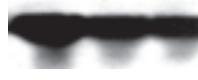

Fig. 4. Seasonal expression of ANR (anthocyanidin reductase) and DkSCPL1 (Diospyros kaki serine carboxypeptidase-like protein 1) in persimmon fruit during fruit development of Chinese pollination-constant, nonastringent (PCNA) 'Luo Tian Tian Shi'; Japanese PCNA 'Suruga'; and pollination-constant, astringent (PCA) 'Kuramitsu'. RNA gel blots are of total RNA $(15 \mu \mathrm{g})$ from persimmon fruit samples taken from June to November.

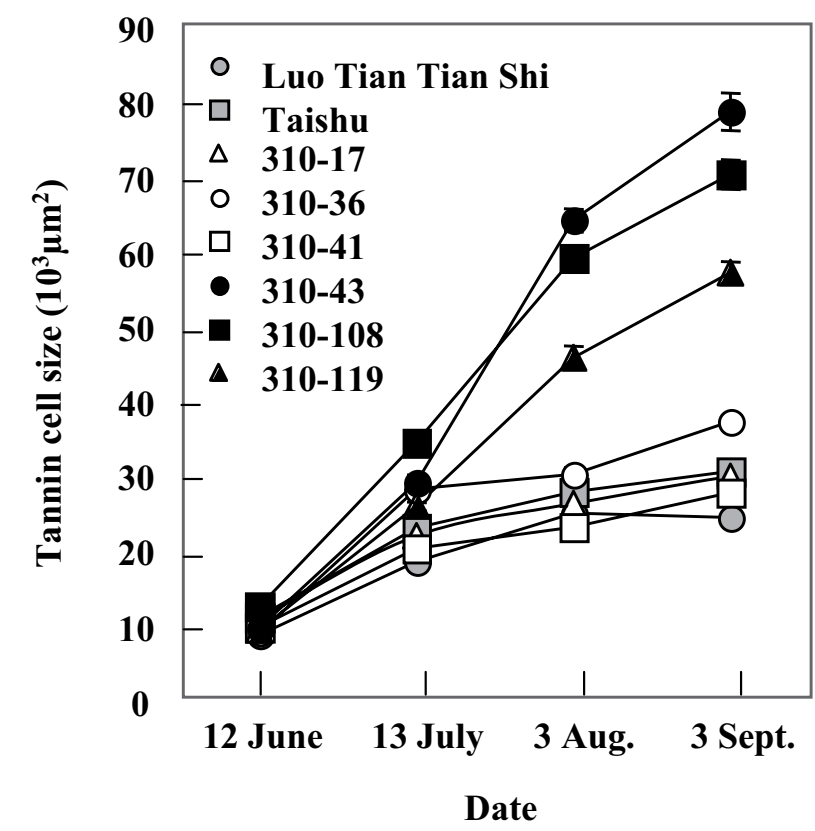

Fig. 5. Changes in tannin cell size measured during fruit development of the $F_{1}$ offspring derived from the cross between 'Luo Tian Tian Shi' and Japanese pollination-constant, nonastringent (PCNA) 'Taishu'. Open symbols are used for PCNA offspring, and filled symbols for non-PCNA offspring.

\section{Discussion}

'Luo Tian Tian Shi' is a PCNA persimmon cultivar that originated in China. In the phylogenic study using AFLP analysis, Kanzaki et al. (2000a) described a close relationship among Japanese PCNA cultivars, whereas 'Luo Tian Tian Shi' proved to be distant. Furthermore, RFLP analysis of 'Luo Tian Tian Shi' with markers that can distinguish Japanese PCNA cultivars from 


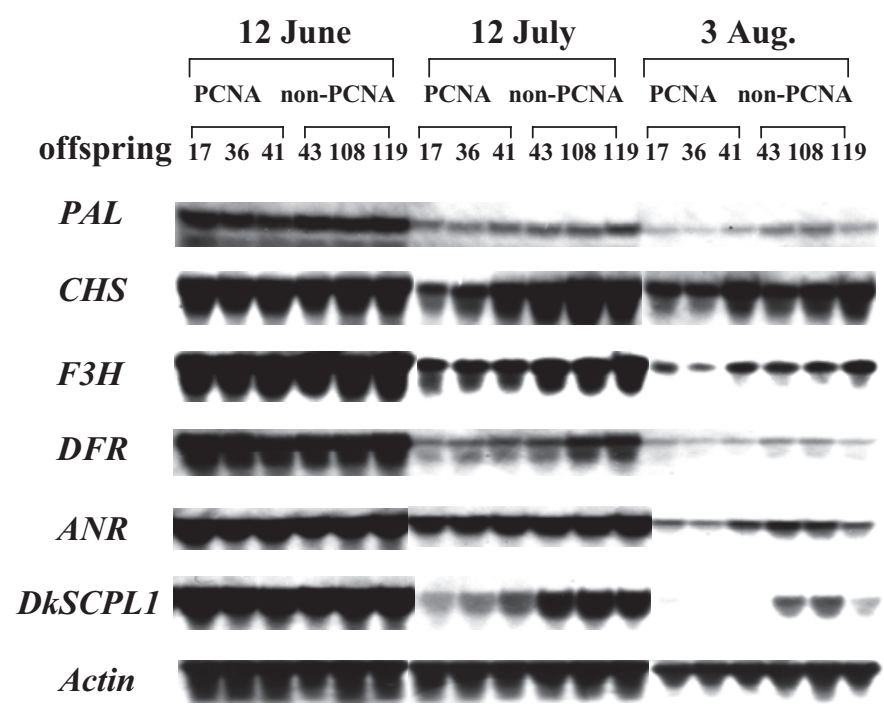

Fig. 6. Seasonal expression of the genes involved in proanthocyanidin biosynthetic pathway in persimmon during fruit development of the $\mathrm{F}_{1}$ offspring derived from the cross between 'Luo Tian Tian Shi' and Japanese pollination-constant, nonastringent (PCNA) 'Taishu'. RNA gel blots are of total RNA (15 $\mu \mathrm{g})$ from persimmon fruit samples taken at 12 June, 12 July, and 3 Aug. Japanese pear (Pyrus pyrifolia) 'Housui' cDNA clone for actin was the probe for a control.

non-PCNA cultivars, demonstrated that 'Luo Tian Tian Shi' has the same RFLP markers as non-PCNA cultivars (Kanzaki et al., 2001). In our previous study, PCNA and non-PCNA individuals were segregated in the $F_{1}$ generation from a cross between PCNA cultivars 'Lou Tian Tian Shi' and 'Taishu', despite the fact that the Japanese PCNA trait is recessive (Ikegami et al., 2004). Additionally, non-PCNA and PCNA-type individuals were also yielded from crosses between 'Luo Tian Tian Shi' and non-PCNA cultivars in $F_{1}$ generation (Ikegami et al., unpublished). These results indicate that 'Luo Tian Tian Shi' has a gene heterozygously for controlling to be PCNA-type and the trait of Chinese PCNA would be dominant.

Astringency of persimmon fruit is due to quantities of condensed tannins, which accumulate into the vacuoles of tannin cells. As proanthocyanindins were synthesized via the flavonoid pathway, we examined gene expressions in the pathway to elucidate the CT accumulation in persimmon fruit at the transcriptional level. Most of the genes involved in flavonoid biosynthetic pathway form the multigene families (Winkel-Shirley, 2001). Thus, the detailed analysis for expression of the genes must be performed with the primer pairs designed for specific genes involved in CT biosynthesis. However, it is difficult to identify all of the gene alleles formed multigene families, as persimmon is hexaploid $(2 \mathrm{n}$ $=6 \mathrm{x}=90$ ). Therefore, we isolated the fragments of these genes involved in flavonoid biosynthetic pathway using degenerate primers from the conserved region of other plants, and designed specific primers for persimmon sequences as described previously (Ikegami et al., 2005). In PCNA cultivars, both Japanese and Chinese 'Luo Tian Tian Shi', tannin cell enlargements and accumulation of CT ceased in early fruit developmental stages. However, expression patterns of the genes involved in flavonoid biosynthesis were different between Japanese and Chinese PCNA cultivars. In the Japanese cultivar, expressions of all of the genes involved in proanthocyanidin synthesis, from $P A L$ to $A N R$, and DkSCPL1 declined along with CT decrease. Thus, the mutation in the Japanese PCNA should be in the upstream or regulatory steps of the flavonoid and phenylpropanoid pathways. However, in 'Luo Tian Tian Shi', gene expression from PAL to ANR, but interestingly not $D k S C P L 1$, remained relatively high despite the termination of tannin cell enlargement. One of the functions of SCPL proteins is proposed to be an acyltransferase accepting 1$O$ - $\beta$-acetal esters as acyl doners, instead of coenzyme A thioesters (Milkiwski and Strack, 2004). The $N$-terminal signal peptide for targeting to the endoplasmic reticulum and the potential $N$-glycosylation site of this SCPL suggests that this acyltransferase is synthesized as pre-protein, and it is transported to the vacuole via the endoplasmic reticulum and Golgi apparatus where the protein is glycosylated (Milkiwski and Strack, 2004; Zuber and Matile 1968). From the deduced amino acid sequence, we identified the potential $N$-terminal signal peptide and four $N$-glycosylation sites in DkSCPL1, which implies the transportation to the vacuole of this protein (Ikegami et al., unpublished). Proanthocyanidins enter the vacuole by transporters, and presumably then the enzymes condense them. According to the proposed structure of condensed tannins in the persimmon fruit (Matsuo and Ito, 1987), they consist of catechin, chatechin-3-O-gallate, gallocatechin, and gallocatechin-3-O-gallate as the repeating units, with molar ration of 1:1:2:2, respectively. Therefore, galloylation of proanthocyanidin must occur in persimmon fruit. However, these biochemical reactions are not well understood. Although glycosylation of flavonoids is regarded as an indispensable signal for vacuolar transport (Fukuchi-Mizutani et al., 2003; Marrs et al., 1995), enzyme for glycosylation of proanthocyadinins is also not identified. A vacuolar transmembrane protein TT12, defined by the A. thaliana $t t 12$ mutant, is involved in transport of proanthocyanidin polymer into the vacuole (Debeaujon et al. 2001). TT12 belongs to the MATE-type transporter family and is thus assumed to recognize some moieties such as glycosyl, acyl, or malonyl moieties (Debeaujon et al., 2001; Kitamura et al., 2004). Considering the structure of condensed tannin in persimmon fruit, formation of flavonoid 3-O- $\beta$-acetal esters may make them transport into vacuole, and acyltransfer by DkSCPL1 may contribute flavonoid 3-O-gallete production in persimmon fruit. However, several experiments should be needed to prove this hypothesis of condensed tannin accumulation into vacuoles of tannin cells in persimmon fruit.

Genes involved in production of secondary metabolites, which have in vivo function to channelize the products into a particular branch of phenylpropanoid or flavonid metabolism, is under strict regulation in plant cells by transcription factors in signal transduction pathways (Endt et al., 2001). As descried previously in this paper, six different types of regulatory elements involved in PA biosynthesis have been cloned and identified (Marles et al., 2003). Mutants of these six regulatory genes shows reduced amount of mRNA levels of ANR (Sagasser et al., 2001; Marles et al., 2003), which is different from continuous transcription levels of $A N R$ in Chinese PCNA fruit in the present study. Therefore, the mutation may relate with the later steps of $A N R$. However, we do not define what kind of mechanisms could explain unchanged transcripts in flavonoid biosynthetic pathway, but decrease in astringency, in Chinese PCNA fruit.

In conclusion, we demonstrated that the Chinese PCNAcultivar behaves similar to PCA cultivar with regard to expression of the genes involved in CT biosynthesis except for DkSCPL1, whereas the termination of tannin cell development occurs as well as the Japanese PCNA cultivar. Continuous transcript levels of ANR and significant decrease in expression of DkSCPL1 might indi- 
cate that the mutation in 'Luo Tain Tian Shi' is closely related to the steps such as sequestration into vacuoles or modification of PAs. Thus, the study of the trait of Chinese PCNA persimmon can provide new information on CT biosynthesis and its regulation in the fruit.

\section{Literature Cited}

Agricultural Research Station. 1912. Investigation on persimmon cultivars (in Japanese). Agr. Res. Sta. (extra) 28:1-46.

Bartel, B. and S.P.T. Matsuda. 2003. Seeing red. Science 299:352353.

Diatchenko, L., Y.F.C. Lau, A.P. Campbell, A. Chenchik, F. Moqadam, B. Huang, S. Lukyanov, K. Lukyanov, N. Gurskaya, E.D. Sverdlov, and P.D. Siebert. 1996. Suppression subtractive hybridization: A method for generating differentially regulated or tissue-specific cDNA probes and libraries. Proc. Natl. Acad. Sci. USA 93:6025-6030

Debeaujon, I., A.J.M. Peeters, K.M. Leon-Kloosterziel, and M. Koornneef. 2001. The TRANSPARENT TESTA12 gene of Arabidopsis encodes a multidrug secondarytransporter-like protein required for flavonoid sequestration in vacuoles of the seed coat endothelium. Plant J. 13:853-871.

Debeaujon, I., N. Nesi, P. Perez, M. Devic, O. Grandjean, M. Caboche, and L. Lepiniec. 2003. Proanthocyanidin-accumulating cells in Arabidopsis testa: Regulation of differentiation and role in seed development. Plant Cell 15:2514-2531.

Endt, D.V., J.W. Kijne, and J. Memelink. 2002. Transcription factors controlling plant secondary metabolism: what regulates the regulators? Phytochemistry 61:107-114.

Fukuchi- Mizutani, M., H. Okuhara, Y. Fukui, M. Nakao, Y. Katsumoto, K. Yonekura-Sakakibara, T. Kusumi, T. Hase, and Y. Tanaka. 2003. Biochemical and molecular characterization of a novel UDP-glucose: Anthocyanin 3'-O-glucosyltransferase, a key enzyme for blue anthocyanin biosynthesis, from Gentian. Plant Physiol. 132:1652-1663.

Ikegami, A., K. Yonemori, A. Sato, M. Yamada, and A. Sugiura. 2004. Segregation of astringency in $\mathrm{F}_{1}$ progenies derived from crosses between pollination-constant, nonastringent persimmon cultivars. HortScience 39:371-374.

Ikegami, A., A. Kitajima, and K. Yonemori. 2005. Inhibition of flavonoid biosynthetic gene expression coincides with loss of astringency in pollination-constant, non-astringent (PCNA)-type persimmon fruit. J. Hort. Sci. Biotechnol. 80:225-228.

Ikeda, I., M. Yamada, A. Kurihara, and T. Nishida. 1985. Inheritance of astringency in Japanese persimmon (in Japanese with English summary). J. Jpn. Soc. Hort. Sci. 54:39-45.

Kanzaki, S., K. Yonemori, A. Sato, M. Yamada, and A. Sugiura. 2000a. Analysis of the genetic relationships among pollination-constant and non-astringent (PCNA) cultivars of persimmon (Diospyros kaki Thunb.) from Japan and China using amplified fragment length polymorphism (AFLP). J. Jpn. Soc. Hort. Sci. 69:665-670.

Kanzaki, S., K. Yonemori, A. Sato, M. Yamada, and A. Sugiura. 2000 b. Evaluation of RFLP analysis for discriminating PCNA genotype in some persimmon cultivars. J. Jpn. Soc. Hort. Sci. 69:702-704.

Kanzaki, S., K. Yonemori, A. Sato, M. Yamada, and A. Sugiura. 2001. Identification of molecular markers linked to the trait of natural astringency loss of Japanese persimmon (Diospyros kaki) fruit. J. Amer. Soc. Hort. Sci. 126:51-55.

Kitanura, S., N. Shikazono, and A. Tanaka. 2004. TRANSPARENT TESTA 19 is involved in the accumulation of both anthocyanins and proanthocyanidins in arabidopsis. Plant J. 37:104-114

Letham, D.S. 1960. The separation of plant cells with ethylenediamineteraacetic acid. Expt. Cell. Res. 21:353-360.

Marles, S.M.A., H. Ray, and M.Y. Gruber. 2003. New perspectives on proanthocyanidin biochemistry and molecular regulation. Phytochemistry 64:367-383.

Marrs, K.A., M.R. Alfenito, A.M. Lloyd, and V. Walbot. 1995. A gluta- thione S-transferase involved in vacuolar transfer encoded by maize gene Bronze-2. Nature 375:397-400.

Matsuo, T. and S. Ito. 1978. The chemical structure of kaki-tannin from immature fruit of the persimmon (Diospyros kaki L.). Agr. Biol. Chem. 42:1637-1643.

Milkowski, C. and D. Strack. 2004. Serine carboxypeptidase-like acyltransferases. Phytochemistry 65:517-524.

Nesi, N., C. Jond, I. Debeaujon, M. Caboche, and L. Lepiniec. 2001. The arabidopsis TT2 gene encodes an R2R3 MYB domain protein that acts as a key determinant for proanthocyanidin accumulation in developing seed. Plant Cell 13:2099-2114.

Park, J., J. Kim, B. Hahn, K. Kim, S. Ha, J. Kim, and Y. Kim. 2004. EST analysis of genes involved in secondary metabolism in Camellia senensis (tea), using suppression subtractive hybridization. Plant Sci. 166:953-961.

Porter,L.J. and J. Woodruffe. 1984. Haemonalysis: the relative astringency of proanthocyanidin polymers. Phytochemistry 23:1268-1270.

Sagasser, M., G. Lu, K. Hahlbrock, and B. Weisshaar. 2001. A. thaliana TRANSPARENT TESTA1 is involved in seed coat development and defines the WIP subfamily of plant zink finger proteins. Genes Dev. 16:138-149.

Sugiura, A. and T. Tomana. 1983. Relationships of ethanol production by seeds of different types of Japanese persimmons and their tannin content. HortScience 18:319-321.

Sugiura, A., H. Harada, and T. Tomana. 1975. Studies on the removability of astringency in Japanese persimmon fruits I. "On-Tree Removal” of astringency by ethanol treatment (in Japanese with English summary). J. Jpn. Soc. Hort. Sci. 44:265-272

Tanner G.I., K.T. Francki, S. Abrahams, J.M. Watson, P.J. Larkin, and A.R. Ashton. 2003. Proanthocyanidin biosynthesis in plants. J. Biol. Chem. 278:31647-31656.

Wan, C.Y. and T.A. Wilkins. 1994. A modified hot borate method significantly enhances the yield of high-quality RNA from cotton (Gossypium hirsutum L.). Anal. Biochem. 223:7-12.

Wang, R. 1982. The origin of 'Luo Tian Tian Shi' (in Chinese). Chinese Fruit Tree 2:16-19.

Wang, R., Y. Yang, and G. Li. 1997. Chinese persimmon germplasm resources. Acta Hort. 436:43-50.

Winkel-Shirley, B. 2001. Flavonoid biosynthesis. A colorful model for genetics, biochemistry, cell biology, and biotechnology. Plant Physiol. 126:485-493.

Winkel-Shirley, B. 2002. Biosynthesis of flavonoids and effects of stress. Curr. Opin. Plant Biol. 5:218-223.

Xie, D.Y., S.B. Sharma, N.L. Paiva, D. Ferreira, and R.A. Dixon. 2003. Role of anthocyanidin reductase, encoded by BANYULS in plant flavonoid biosynthesis. Science 299:396-399.

Yamada, M. 1993. Persimmon breeding in Japan. Jpn. Agr. Res. Quarterly 27:33-37.

Yamada, M., H. Yamane, and Y. Ukai. 1994. Genetic analysis of Japanese persimmon fruit weight. J. Amer. Soc. Hort. Sci. 199:1298-1302.

Yamada, M. and A. Sato. 2002. Segregation for fruit astringency type in progenies derived from crosses of 'Nishimurawase' $\mathbf{x}$ pollination constant non-astringent genotypes in oriental persimmon (Diospyros kaki Thunb.). Scientia Hort. 92:107-111.

Yonemori, K. and J. Matsushima. 1985. Property of development of the tannin cells in non-astringent type fruits of Japanese persimmon (Diospyros kaki) and its relationship to natural astringency (in Japanese with English summary). J. Jpn. Soc. Hort. Sci. 54:201-208.

Yonemori, K. and J. Mastushima. 1987. Changes in tannin cell morphology with growth and development of Japanese persimmon fruit. J. Amer. Soc. Hort. Sci. 112:818-821.

Yonemori, K., A. Sugiura, and M. Yamada. 2000. Persimmon genetics and breeding. Plant Breeding Rev. 19:191-225.

Zuber, H. and P.H. Matile. 1968. Acid carboxypeptidase: Their occurrence in plants, intracellular distribution and possible function. $\mathrm{Z}$. Naturforsch. 23b:663-665 